\title{
Re-biopsy of partially sampled thin melanoma impacts sentinel lymph node sampling as well as surgical margins
}

\author{
Evan S Weitman*,1, Matthew C Perez ${ }^{1}$, Daniel Lee ${ }^{1}$, Youngchul Kim¹, William Fulp ${ }^{1}$, \\ Vernon K Sondak ${ }^{1}$, Amod A Sarnaik ${ }^{1}$, Ricardo J Gonzalez ${ }^{1}$, Carl W Cruse ${ }^{1}$, Jane L Messina ${ }^{1}$ \\ \& Jonathan S Zager ${ }^{1}$ \\ ${ }^{1}$ Moffitt Cancer Center, Department of Cutaneous Oncology, 12902 Magnolia Drive, Tampa, FL 33602, USA \\ *Author for correspondence: evanweitman@gmail.com
}

\begin{abstract}
Aim: To assess the impact of re-biopsy on partially sampled melanoma in situ (MIS), atypical melanocytic proliferation (AMP) and thin invasive melanoma. Materials \& methods: We retrospectively identified cases of re-biopsied partially sampled neoplasms initially diagnosed as melanoma in situ, AMP or thin melanoma (Breslow depth $\leq 0.75 \mathrm{~mm}$ ). Results \& conclusion: Re-biopsy led to sentinel lymph node biopsy (SLNB) in $18.3 \%$ of cases. No patients upstaged from AMP or MIS had a positive SLNB. One out of nine (11.1\%) initially diagnosed as a thin melanoma $\leq 0.75 \mathrm{~mm}$, upstaged with a re-biopsy, had a positive SLNB. After rebiopsy $8.5 \%$ underwent an increased surgical margin. Selective re-biopsy of partially sampled melanoma with gross residual disease can increase the accuracy of microstaging and optimize treatment regarding surgical margins and SLNB.
\end{abstract}

First draft submitted: 9 December 2018; Accepted for publication: 28 February 2019; Published online: 26 April 2019

Keywords: atypical melanocytic proliferation • Breslow depth • melanoma • melanoma in situ • sentinel lymph node biopsy • surgical margin

The incidence of cutaneous melanoma has been increasing over the past several decades, with an estimated 178,560 new melanoma cases and 9320 deaths due to melanoma in 2018 in the USA [1]. The majority of melanoma patients initially present with localized disease, with thin melanomas, defined as $\leq 1 \mathrm{~mm}$ in thickness, accounting for approximately $70 \%$ of new melanoma cases [2]. 5-year melanoma-specific survival rates are excellent for stages I and II disease (98 and 90\%, respectively); however, survival drops dramatically for melanoma patients with stages III and IV disease (77 and 10\%, respectively) [3].

Accurate diagnosis is of paramount importance in the initial surgical management of cutaneous melanoma [4,5]. Primary tumor characteristics, primarily depth of invasion (Breslow thickness), guide clinicians in determining appropriate surgical margins of excision for the primary tumor as well as the decision to surgically stage the regional nodal basin [6,7]. Therefore, an initial biopsy for suspected cutaneous melanoma optimally includes the entire lesion to ensure accurate staging of the primary tumor and minimize sampling error [8].

Current guidelines from the National Comprehensive Cancer Network recommend an excisional biopsy of clinically suspicious cutaneous lesions with 1-3 mm margins if feasible [9]. There are a variety of biopsy techniques utilized by both dermatologists as well as surgeons, including shave biopsy, punch biopsy of varying sizes and narrow margin excisional biopsy [10-12]. Shave biopsy has been demonstrated to be safe and accurate for diagnosis of thin melanomas [13]. Excisional biopsy usually (but not always) completely samples the lesion; however, it is more time intensive and cumbersome than a shave or punch biopsy [14].

While prior studies have not demonstrated a correlation between biopsy technique and recurrence or survival, the impact of re-biopsy for partially sampled melanoma with gross residual disease has not been previously studied [15]. It has, however, been shown that partial sampling of lesions can lead to inaccurate staging, additional surgical procedures and increased anxiety for patients $[16,17]$. Clinical judgment should be exercised on initial assessment of larger cutaneous lesions with concerning features such as asymmetry, border irregularity, color changes or a history

Future Medicine 
of enlarging diameter $[18,19]$. The situation can arise where a lesion with such features has undergone suboptimal biopsy and may underestimate the true depth of the lesion. Re-biopsy can be utilized in this clinical scenario to rule out a deeper invasive lesion.

In this study, we reviewed our experience with re-biopsy of clinically concerning, residual cutaneous melanocytic neoplasms up to $0.75 \mathrm{~mm}$ in depth prior to surgical treatment and assessed how often surgical management was modified due to this practice. We also included partially sampled lesions initially diagnosed as atypical melanocytic proliferations (AMP) or melanoma in situ (MIS), as these can represent inadequately sampled invasive melanomas and hence re-biopsy of a residual pigmented lesion could potentially upstage these lesions and alter management [20]. We excluded patients with positive biopsy edges or residual pigmentation; however, who did not undergo re-biopsy.

\section{Methods}

After obtaining Institutional Review Board approval, a retrospective review was conducted involving patients referred to Moffitt Cancer Center between 2006 and 2017 who underwent re-biopsy of a partially sampled lesion, which was initially read as AMP, MIS or invasive melanoma with a depth limited to $\leq 0.75 \mathrm{~mm}$. All re-biopsies were performed due to the clinician identifying a residual pigmented lesion on examination. Repeat biopsies were typically performed with the intention of a complete punch excision of all residual pigment.

At Moffitt, sentinel lymph node biopsy (SLNB) is typically performed for invasive melanomas $>0.75 \mathrm{~mm}$ in depth per seventh edition American Joint Committee on Cancer (AJCC; now $\geq 0.8 \mathrm{~mm}$ per eighth edition AJCC). Therefore, we only included patients with invasive melanoma of $\leq 0.75 \mathrm{~mm}$ on initial biopsy, as these patients would not routinely have undergone SLNB without an additional biopsy demonstrating an increased thickness. SLNB was performed according to techniques previously described [21,22]. Demographic and clinical information as well as primary tumor pathology were captured, and surgical outcome data including nodal status and final pathology on wide excision specimens were reviewed.

AMP and MIS are typically excised with a $0.5 \mathrm{~cm}$ surgical resection margins. Invasive cutaneous melanomas up to $0.9 \mathrm{~mm}$ in thickness are resected with a $1 \mathrm{~cm}$ margin. For lesions between 1.0 and $1.9 \mathrm{~mm}$ in thickness, we selectively utilize $1-2 \mathrm{~cm}$ wide excision margins [23]. For lesions with a thickness $\geq 2 \mathrm{~mm}$, we routinely utilize a $2 \mathrm{~cm}$ margin. Occasionally, margins of excision are modified for functional or aesthetic considerations; however, when feasible, these excision guidelines are utilized.

All cases were reviewed by a dermatopathologist at Moffitt Cancer Center. Primary tumor characteristics including Breslow thickness and ulceration were assessed. Lymph nodes obtained during SLNB were assessed using our previously published protocol [2]. Patient characteristics were summarized using descriptive statistics including mean and range for continuous measures and proportions and frequencies for categorical measures.

\section{Results}

A total of 71 patients were included in the study. Table 1 shows the descriptive statistics for basic demographics, biopsy data and surgical outcomes. The mean age was 65 years old, with an approximately equal number of men $(50.7 \%)$ and women (49.3\%). Tumors were most commonly found on the extremities, accounting for $62 \%$ of cases. The most common type of initial biopsy was a shave biopsy (64.8\%), followed by punch biopsy (33.8\%). Repeat biopsies of the residual pigmented lesion present at consultation at Moffitt were most commonly punch biopsies (85.9\%). On initial biopsy, most cases (70.4\%) were nonulcerated invasive thin melanomas. AMP and MIS accounted for 7 and $16 \%$ of patients, respectively.

Of the 71 patients in this series who underwent repeat biopsy of apparent residual tumor, five patients $(7.0 \%)$ had no residual tumor identified in the re-biopsy specimen, while 13 patients $(18.3 \%)$ were proven to have an invasive melanoma $>0.75 \mathrm{~mm}$ in thickness. Four of the five patients without residual tumor identified on re-biopsy were initially staged as MIS on their referral biopsy. One of those five patients without residual tumor identified on re-biopsy was initially staged as a $0.2 \mathrm{~mm}$ invasive melanoma. Pathologic review of re-biopsy specimens for these five patients demonstrated benign pigmented lesions, such as melanocytic hyperplasia. MIS was identified on the wide excision specimen in two of these cases, despite no residual melanoma identified on re-biopsy.

Regarding the 13 patients who were upstaged after re-biopsy to an invasive melanoma $>0.75 \mathrm{~mm}$ in thickness, one initially presented with a referral biopsy of MIS, two initially presented with a referral biopsy of AMP and ten initially presented with a referral biopsy of invasive melanoma $<0.76 \mathrm{~mm}$. All 13 of these patients underwent SLNB (Table 2). One of the 13 patients failed to localize a sentinel node during lymphoscintigraphy and was followed by serial ultrasound examinations of the at-risk regional nodal basin. Of the 12 patients who underwent 
Table 1. Patient characteristics.

Patient characteristic

Age

Gender:

- Female

- Male

Tumor site:

- Extremity

- Trunk

- Head \& neck

Initial biopsy type:

- Punch

- Shave

- Excisional

Initial Breslow depth:

- AMP

- In situ

- Invasive: $0-0.75$

Initial Breslow depth invasive (mean)

Ulceration:

- No

- Yes

Additional biopsy type:

- Punch

- Shave

- Excisional

AMP: Atypical melanocytic proliferation.
Value

$65(12 ; 90)$

$35(49 \%)$

$36(51 \%)$

$44(62 \%)$

$19(27 \%)$

$8(11 \%)$

$24(34 \%)$

$46(65 \%)$

$1(1 \%)$

$5(7 \%)$

$16(23 \%)$

$50(70 \%)$

0.5

$48(96 \%)$

$2(4 \%)$

$61(86 \%)$

$7(10 \%)$

$3(4 \%)$

Table 2. Biopsy results, sentinel lymph node biopsy status and margin status by initial referral biopsy stage.

\begin{tabular}{|c|c|c|c|c|}
\hline \multicolumn{5}{|c|}{ MIS } \\
\hline Initial biopsy depth & $\begin{array}{l}\text { Cumulative depth after } \\
\text { re-biopsy }\end{array}$ & $\begin{array}{l}\text { Cumulative depth after wide } \\
\text { excision }\end{array}$ & SLNB status & $\begin{array}{l}\text { Change in surgical margins due } \\
\text { to re-biopsy }\end{array}$ \\
\hline \multirow[t]{3}{*}{ MIS (16) } & Still MIS (13) & Still MIS (12) & $0 / 1(+)$ & $3 / 16(19 \%)$ \\
\hline & T1a (2) & T1a (2) & & \\
\hline & $\mathrm{T} 2 \mathrm{a}(1)$ & T2a (2) & & \\
\hline \multicolumn{5}{|c|}{ AMP } \\
\hline Initial biopsy depth & $\begin{array}{l}\text { Cumulative depth after } \\
\text { re-biopsy }\end{array}$ & $\begin{array}{l}\text { Cumulative depth after wide } \\
\text { excision }\end{array}$ & SLNB status & $\begin{array}{l}\text { Change in surgical margins due } \\
\text { to re-biopsy }\end{array}$ \\
\hline \multirow[t]{3}{*}{ AMP (5) } & Still AMP (3) & Still AMP (3) & $0 / 2(+)$ & $2 / 5(40 \%)$ \\
\hline & $\mathrm{T} 2 \mathrm{a}(1)$ & T2a (1) & & \\
\hline & T3a (1) & T3a (1) & & \\
\hline \multicolumn{5}{|c|}{ Thin invasive melanoma } \\
\hline Initial biopsy depth & $\begin{array}{l}\text { Cumulative depth after } \\
\text { re-biopsy }\end{array}$ & $\begin{array}{l}\text { Cumulative depth after wide } \\
\text { excision }\end{array}$ & SLNB status & $\begin{array}{l}\text { Change in surgical margins due } \\
\text { to re-biopsy }\end{array}$ \\
\hline T1a (48) & Still T1a (39) & Still T1a (38) & $\begin{array}{l}1 / 9(+) \\
\text { SLNB aborted in } 1 \text { case due to } \\
\text { nonlocalization }\end{array}$ & $1 / 50(2 \%)$ \\
\hline \multirow[t]{4}{*}{$\mathrm{T} 1 \mathrm{~b}(2)$} & $\mathrm{T} 1 \mathrm{~b}(6)$ & $\mathrm{T} 1 \mathrm{~b}(5)$ & & \\
\hline & $\mathrm{T} 2 \mathrm{a}(3)$ & T2a (5) & & \\
\hline & $\mathrm{T} 2 \mathrm{~b}(1)$ & $\mathrm{T} 2 \mathrm{~b}(1)$ & & \\
\hline & T3a (1) & T3a (1) & & \\
\hline
\end{tabular}

AMP: Atypical melanocytic proliferation; MIS: Melanoma in situ; SLNB: Sentinel lymph node biopsy. 
successful SLNB, one had a positive sentinel node for an overall $8.3 \%$ positivity rate. None of the patients initially diagnosed as MIS or AMP went on to have a positive SLNB. One of nine (11.1\%) patients with an initial diagnosis of thin melanoma $\leq 0.75 \mathrm{~mm}$ had a positive SLNB.

Despite the performance of repeat biopsies, two patients were found to have unrecognized invasive melanoma $>0.75 \mathrm{~mm}$ in the wide excision specimen. One had an initial referral diagnosis of $0.5 \mathrm{~mm}$, and re-biopsy demonstrated a $0.5 \mathrm{~mm}$ melanoma while the wide excision specimen ultimately revealed a $1.1 \mathrm{~mm}$ melanoma. The other patient had an initial referral diagnosis of MIS, and re-biopsy demonstrated MIS while the wide excision specimen ultimately revealed an invasive $1.4 \mathrm{~mm}$ melanoma. Those two patients elected for ultrasound surveillance of the lymphatic basin and remain well without evidence of nodal recurrence at this time. On multivariate logistic regression analysis, males were more likely to have had SLNB performed due to re-biopsy compared with females (OR [95\% CI]: 2.177 [0.574, 9.381]), although this was not statistically significant. In the model, initial biopsy type was also not a statistically significant indicator of SLNB being performed due to re-biopsy.

Among the five patients initially characterized on referral biopsy as AMP, after re-biopsy of the residual lesion one was reclassified as T2a and one reclassified as T3a. Overall, six of $71(8.5 \%)$ patients underwent an increased width of excision due to re-biopsy (prereferral diagnoses were: MIS 3/16 [19\%], AMP 2/5 [40\%], thin melanoma 1/50 [2\%]; Table 2). All six patients who underwent a change in surgical margins had tumors arising on an extremity. Following the repeat biopsy of these lesions in clinic prior to definitive surgery, 15/71 (21.1\%) cases demonstrated no residual tumor in the final wide excision specimen.

Overall, our results demonstrate $96 \%$ final T stage accuracy after re-biopsy. Of those not accurately staged after re-biopsy, two patients were upstaged from a T1a to T2a invasive melanoma, and one patient was upstaged from MIS to a T2a invasive melanoma.

\section{Discussion}

In this study, we aimed to address the clinical situation in which a significant portion of a pigmented lesion remains visible on the patient's skin after an initial biopsy. When performing a biopsy on a larger cutaneous lesion, shave or punch biopsies are often inadequate in removing all gross visible tumor. We hypothesized that in such cases there may be a higher likelihood of clinically significant underestimation of the true extent of disease than in cases with microscopically positive biopsy edges but no visible residual lesion. The impact of re-biopsy of a partially sampled cutaneous melanoma with gross residual disease has not previously been addressed in the literature.

We retrospectively identified 71 cases of re-biopsied partially sampled AMP, MIS or thin melanoma $(\leq 0.75 \mathrm{~mm})$ to evaluate how often re-biopsy of the residual pigmented lesion resulted in tumor upstaging. The cut-off depth of $0.75 \mathrm{~mm}$ was used based on our institutional practice of performing SLNB for cutaneous melanoma $>0.75 \mathrm{~mm}$ in depth. Notably, after re-biopsy $18 \%$ of patients were upstaged to $>0.75 \mathrm{~mm}$, and hence identified as appropriate for SLNB. We had an $8.3 \%$ SLNB positivity rate in our patients who were upstaged after re-biopsy to $>0.75 \mathrm{~mm}$. Despite our small sample size (only 13 patients underwent SLNB after re-biopsy), our findings are highly consistent with previously published findings of an $8.4 \%$ SLNB positivity rate for thin melanomas $>0.75 \mathrm{~mm}$ in depth [2]. None of these patients would have routinely undergone SLNB at the initial surgery without an additional biopsy.

A complete biopsy of a suspicious lesion typically provides sufficient information to decide on proper surgical margins and the appropriateness of lymph node sampling. In the setting of partially sampled lesions, the depth and degree of invasiveness can sometimes be underestimated. Once a wide local excision is completed, the true depth of the lesion is identified. The option to perform an SLNB is still available, but the lymphoscintigraphy loses some of its specificity. Performing SLNB in delayed fashion after wide excision can potentially lead to decreased sensitivity, due to disruption and damage of lymphatics directly draining the site of the primary lesion [24]. On the other hand, it must be conceded that in a series of 71 patients undergoing re-biopsy of suspected residual melanoma, only one sentinel node-positive patient was identified, five cases had no residual lesion in the re-biopsy specimen and two patients with melanomas thick enough to warrant SLNB but were not identified as such even by re-biopsy.

The precise impact of re-biopsy on excision margins will depend on the algorithm used for determining excision margins. While guidelines for MIS, for example, call for at least a $0.5 \mathrm{~cm}$ excision margin, many surgeons use a 1.0 $\mathrm{cm}$ margin where feasible, especially in the presence of visible residual disease. In our 16 patients with MIS and residual pigmented lesion, if no re-biopsy and a $0.5 \mathrm{~cm}$ margin would have been done, 4 patients would have been found to have been undertreated due to the actual presence of invasive melanoma - and re-biopsy identified this in all but 1 case. Conversely, if all these patients had been treated with a $1 \mathrm{~cm}$ margin without re-biopsy, in only one case would the finding of an invasive melanoma $>1.00 \mathrm{~mm}$ in the wide excision have called into question the 
adequacy of the excision margin. In most cases the lesion will be completely excised regardless of a 0.5 - versus 1.0 $\mathrm{cm}$ margin; however, this can potentially affect locoregional control, particularly in the setting of lymphovascular invasion.

Surgical margins were not significantly impacted for re-biopsied invasive melanomas (2\%); however, this was not surprising as T2 lesions can often be managed appropriately with a $1 \mathrm{~cm}$ surgical margin. The management of partially sampled invasive melanomas was significantly impacted by re-biopsy regarding the decision to perform an SLNB. Only one of these upstaged patients ultimately had metastatic melanoma identified on SLNB; however, $10 / 50$ patients $(20 \%)$ underwent SLNB who otherwise would not have on their initial surgery.

Another conundrum is what to do about a diagnosis of AMP with residual pigmented lesion, where the pathologist may well be underestimating the gravity of the lesion. Our data support the fact that some of these lesions will be found to represent MIS or even invasive melanoma. Our series included five such cases, and two were found to be intermediate thickness melanomas; both of which merited SLNB and an increased wide excision margin. A limitation of this study is the sample size of only five patients in the AMP group. Future studies with a larger sample size of partially sampled AMP would be valuable to potentially reinforce our findings. Narrow excisional biopsy is certainly a reasonable option in this clinical scenario. Our clinical series features partially sampled lesions where AMP was demonstrated on initial biopsy but the residual lesion was concerning enough to merit a re-biopsy in clinic due to its suspicion for harboring an invasive melanoma. Our $40 \%$ upstage value for AMP confirms that clinical acumen can help guide when to re-biopsy suspicious lesions. This information is important and useful, as it can help guide the clinician to raise their index of suspicion when initial biopsy demonstrates an AMP but the lesion appears more consistent with an invasive melanoma.

Clearly, the potential for under or overtreatment of these lesions is great, and so use of re-biopsy should be carefully considered. A strict rule is probably not appropriate to guide decision making in these cases and some degree of clinical discretion should be utilized. For instance, in the setting of larger, clinically worrisome lesions it seems more likely that partial biopsy prior to wide excision would potentially underestimate the true depth of disease.

Most initial biopsies were performed by shave biopsy and most repeat biopsies were performed by punch biopsy, but not surprisingly, we did not find any correlation between upstaging of the lesion upon re-biopsy and biopsy type $[13,16,25]$. It should be stressed that since our cohort of patients had gross residual disease, our findings clearly will not be directly applicable to those patients with a positive microscopic margin on initial biopsy but no visible residual disease on clinical examination. We did not have the initial lesion size available to review, which could have potentially facilitated better characterization of high- versus low-risk partially sampled lesions. Additionally, it should be noted that the patients in our series were typically initially biopsied prior to arrival at our institution from a variety of different practices, and it is subsequently difficult to comment on the indications, techniques and decision making involved in the initial biopsy. This clinical scenario of partially sampled lesions is sometimes the manifestation of a suboptimal initial workup. Nonetheless, results from this series can potentially help guide clinicians when they find themselves in this situation.

\section{Conclusion}

Re-biopsy of partially sampled premalignant lesions and thin melanomas with gross residual disease can impact the decision to perform SLNB as well as the extent of surgical margins. Overall, our results demonstrate $96 \% \mathrm{~T}$ staging accuracy after re-biopsy of a clinically suspicious residual pigmented lesion [26]. We therefore would recommend consideration of re-biopsy of partially sampled melanoma in the setting of concerning residual disease in any case where it might be expected to potentially alter surgical management of the primary lesion or regional nodes.

\section{Future perspective}

Melanoma treatment has evolved dramatically over the past decade due to the introduction of targeted therapies, such as BRAF-MEK inhibitors, and immunotherapy. These innovative treatment modalities have had their greatest impact on the management of locally advanced and metastatic melanoma. Yet, the majority of melanoma presents as early stage disease and clinical decision making is initially driven by adequate biopsy to facilitate an accurate diagnosis. Surgical resection is still the mainstay of treatment for localized cutaneous melanoma. However, in the aftermath of the MSLT-2 trial [27], melanoma surgeons have become increasingly selective in when to perform completion lymph node dissection. In the next 5-10 years, as the biology of lymphatic metastasis is further elucidated and as targeted therapy becomes increasingly effective, the use of completion lymph node dissection 
in melanoma may completely disappear. Despite these changing algorithms, the prognostic value of SLNB will likely remain durable and should remain in the surgical armamentarium to provide accurate staging for melanoma. Similarly, early and complete biopsy of suspicious lesions will likely remain the gold standard for the initial diagnosis of melanoma.

\section{Executive summary}

- Partially sampled pigmented lesions containing melanoma in situ (MIS), atypical melanocytic proliferation (AMP) and early invasive melanoma $(\leq 0.75 \mathrm{~mm})$ can potentially harbor a deeper invasive melanoma.

- Re-biopsy of partially sampled MIS, AMP and early invasive melanoma can be appropriate in the right clinical context, specifically in the setting of larger pigmented lesions with concerning residual disease.

- In our case series we demonstrate that re-biopsy of partially sampled MIS, AMP and early invasive melanoma $(\leq 0.75 \mathrm{~mm}$ ) led to sentinel lymph node biopsy (SLNB) in $18.3 \%$ of cases which otherwise would not have undergone any lymph node sampling.

- Of note, the SLNB positivity rate was $0 \%$ in cases originally identified as either MIS or AMP on initial biopsy. The SLNB positivity rate was $11 \%$ in cases originally identified as early invasive melanoma $(\leq 0.75 \mathrm{~mm})$ on initial biopsy.

- Surgical margin status was impacted in $8.5 \%$ of cases due to re-biopsy.

- Selective re-biopsy of partially sampled melanoma with gross residual disease can increase the accuracy of microstaging and optimize treatment regarding surgical margins and SLNB.

\section{Financial \& competing interests disclosure}

The authors have no relevant affiliations or financial involvement with any organization or entity with a financial interest in or financial conflict with the subject matter or materials discussed in the manuscript. This includes employment, consultancies, honoraria, stock ownership or options, expert testimony, grants or patents received or pending, or royalties.

No writing assistance was utilized in the production of this manuscript.

\section{Ethical conduct of research}

The authors state that they have obtained appropriate Institutional Review Board approval or have followed the principles outlined in the Declaration of Helsinki for all human or animal experimental investigations. In addition, for investigations involving human subjects, informed consent has been obtained from the participants involved.

\section{Open access}

This work is licensed under the Attribution-NonCommercial-NoDerivatives 4.0 Unported License. To view a copy of this license, visit http://creativecommons.org/licenses/by-nc-nd/4.0/

\section{References}

Papers of special note have been highlighted as: $\bullet$ of interest

1. Siegel RL, Miller KD, Jemal A. Cancer statistics, 2018. CA Cancer J. Clin. 68(1), 7-30 (2018).

2. Han D, Yu D, Zhao X et al. Sentinel node biopsy is indicated for thin melanomas $\geq 0.76 \mathrm{~mm}$. Ann. Surg. Oncol. 19(11), 3335-3342 (2012).

- Particularly important paper as it helps in establishing $0.76 \mathrm{~mm}$ as a threshold for sentinel lymph node biopsy in the setting of invasive melanoma.

3. Gershenwald JE, Scolyer RA, Hess KR et al. Melanoma staging: evidence-based changes in the American Joint Committee on Cancer eighth edition cancer staging manual. CA. Cancer J. Clin. 67(6), 472-492 (2017).

4. Silverstein D, Mariwalla K. Biopsy of the pigmented lesions. Dermatol. Clin. 30(3), 435-443 (2012).

5. Tran KT, Wright NA, Cockerell CJ. Biopsy of the pigmented lesion - when and how. J. Am. Acad. Dermatol. 59(5), 852-871 (2008).

6. Amin MB, Greene FL, Edge SB et al. The Eighth Edition AJCC Cancer Staging Manual: continuing to build a bridge from a population-based to a more 'personalized' approach to cancer staging. CA Cancer J. Clin. 67(2), 93-99 (2017).

7. Breslow A. Thickness, cross-sectional areas and depth of invasion in the prognosis of cutaneous melanoma. Ann. Surg. 172(5), 902-908 (1970).

8. Ng JC, Swain S, Dowling JP, Wolfe R, Simpson P, Kelly JW. The impact of partial biopsy on histopathologic diagnosis of cutaneous melanoma: experience of an Australian tertiary referral service. Arch. Dermatol. 146(3), 234-239 (2010).

9. National Comprehensive Cancer Network. Cutaneous Melanoma Version 2, (2019). www.nccn.org 
10. Roses DF, Ackerman AB, Harris MN, Weinhouse GR, Gumport SL. Assessment of biopsy techniques and histopathologic interpretations of primary cutaneous malignant melanoma. Ann. Surg. 189(3), 294-297 (1979).

11. Pickett H. Shave and punch biopsy for skin lesions. Am. Fam. Physician 84(9), 995-1002 (2011).

12. Ng PC, Barzilai DA, Ismail SA, Averitte RL Jr, Gilliam AC. Evaluating invasive cutaneous melanoma: is the initial biopsy representative of the final depth? J. Am. Acad. Dermatol. 48(3), 420-424 (2003).

13. Zager JS, Hochwald SN, Marzban SS et al. Shave biopsy is a safe and accurate method for the initial evaluation of melanoma. J. Am. Coll. Surg. 212(4), 454-460; discussion 60-2 (2011).

14. Stell VH, Norton HJ, Smith KS, Salo JC, White RL Jr. Method of biopsy and incidence of positive margins in primary melanoma. Ann. Surg. Oncol. 14(2), 893-898 (2007).

15. Molenkamp BG, Sluijter BJ, Oosterhof B, Meijer S, van Leeuwen PA. Non-radical diagnostic biopsies do not negatively influence melanoma patient survival. Ann. Surg. Oncol. 14(4), 1424-1430 (2007).

16. Mills JK, White I, Diggs B, Fortino J, Vetto JT. Effect of biopsy type on outcomes in the treatment of primary cutaneous melanoma. Am. J Surg. 205(5), 585-590; discussion 90 (2013).

17. Pflugfelder A, Weide B, Eigentler TK et al. Incisional biopsy and melanoma prognosis: facts and controversies. Clin. Dermatol. 28(3), 316-318 (2010).

18. Friedman RJ, Rigel DS, Kopf AW. Early detection of malignant melanoma: the role of physician examination and self-examination of the skin. CA Cancer J. Clin. 35(3), 130-151 (1985).

19. Daniel Jensen J, Elewski BE. The ABCDEF rule: combining the 'ABCDE Rule' and the 'Ugly Duckling Sign' in an effort to improve patient self-screening examinations. J. Clin. Aesthet. Dermatol. 8(2), 15 (2015).

20. Cohen LM, Hodge SJ, Owen LG, Callen JP. Atypical melanocytic nevi. clinical and histopathologic predictors of residual tumor at reexcision. J. Am. Acad. Dermatol. 27(5 Pt 1), 701-706 (1992).

21. Ross MI, Reintgen D, Balch CM. Selective lymphadenectomy: emerging role for lymphatic mapping and sentinel node biopsy in the management of early stage melanoma. Semin. Surg. Oncol. 9(3), 219-223 (1993).

22. Morton DL, Wen DR, Wong JH et al. Technical details of intraoperative lymphatic mapping for early stage melanoma. Arch. Surg. 127(4), 392-399 (1992).

23. Doepker MP, Thompson ZJ, Fisher KJ et al. Is a wider margin $(2 \mathrm{~cm}$ vs $1 \mathrm{~cm})$ for a $1.01-2.0 \mathrm{~mm}$ melanoma necessary? Ann. Surg. Oncol. 23(7), 2336-2342 (2016).

24. Gannon CJ, Rousseau DL Jr, Ross MI et al. Accuracy of lymphatic mapping and sentinel lymph node biopsy after previous wide local excision in patients with primary melanoma. Cancer 107(11), 2647-2652 (2006).

25. Hieken TJ, Hernandez-Irizarry R, Boll JM, Jones Coleman JE. Accuracy of diagnostic biopsy for cutaneous melanoma: implications for surgical oncologists. Int. J. Surg. Oncol. 2013, 196493 (2013).

26. Egnatios GL, Dueck AC, Macdonald JB et al. The impact of biopsy technique on upstaging, residual disease, and outcome in cutaneous melanoma. Am. J. Surg. 202(6), 771-777; discussion 7-8 (2011).

- Addresses the issue of residual disease after variable biopsy techniques for cutaneous melanoma and helped set the stage for future studies such as our own.

27. Faries MB, Thompson JF, Cochran AJ et al. Completion dissection or observation for sentinel-node metastasis in melanoma. N. Engl. J. Med. 376(23), 2211-2222 (2017). 